\title{
Critical behavior of intercalated quasi-van der Waals ferromagnet $\mathrm{Fe}_{0.26} \mathrm{TaS}_{2}$
}

\author{
Chenhui Zhang $\odot,{ }^{1, *}$ Ye Yuan, ${ }^{1, *}$ Mao Wang, ${ }^{2,3}$ Peng Li,,${ }^{1}$ Junwei Zhang, ${ }^{1}$ Yan Wen, ${ }^{1}$ \\ Shengqiang Zhou $\odot,{ }^{2}$ and Xi-Xiang Zhang $\oplus^{1, \dagger}$ \\ ${ }^{1}$ Physical Science and Engineering Division, King Abdullah University of Science and Technology (KAUST), \\ Thuwal 23955-6900, Saudi Arabia \\ ${ }^{2}$ Helmholtz-Zentrum Dresden-Rossendorf, Institute of Ion Beam Physics and Materials Research, \\ Bautzner Landstrasse 400, D-01328 Dresden, Germany \\ ${ }^{3}$ Institut für Angewandte Physik (IAP), Technische Universität Dresden, D-01062 Dresden, Germany
}

(Received 16 August 2019; published 5 November 2019)

\begin{abstract}
In the present work, single-crystalline quasi-van der Waals ferromagnet $\mathrm{Fe}_{0.26} \mathrm{TaS}_{2}$ was successfully synthesized with Fe atoms intercalated at ordered positions between $\mathrm{TaS}_{2}$ layers. Its critical behavior was systematically studied by measuring the magnetization around ferromagnetic to paramagnetic phase transition temperature, $T_{C} \sim 100.7 \mathrm{~K}$, under different magnetic fields. The critical exponent $\beta$ for the spontaneous magnetization below $T_{C}, \gamma$ for the inverse initial susceptibility above $T_{C}$, and $\delta$ for the magnetic isotherm at $T_{C}$ were determined with modified Arrott plots, the Kouvel-Fisher method, the Widom scaling law, and critical isotherm analysis, and found to be the following values: $\beta=0.459(6), \gamma=1.205(11)$, and $\delta=3.69(1)$. The obtained critical exponents are self-consistent and follow the scaling equation, indicating the reliability and intrinsicality of these parameters. A close analysis within the framework of renormalization group theory reveals that the spin coupling inside $\mathrm{Fe}_{0.26} \mathrm{TaS}_{2}$ crystal is of the three-dimensional Heisenberg $(\{d: n\}=\{3: 3\})$ type with long-range magnetic interaction and that the exchange interaction decays with distance as $J(r) \sim r^{-4.71}$.
\end{abstract}

DOI: 10.1103/PhysRevMaterials.3.114403

\section{INTRODUCTION}

The discovery of the magnetic van der Waals (vdW) crystals that present intrinsic ferromagnetism even down to the monolayer limit, such as $\mathrm{Cr}_{2} \mathrm{Ge}_{2} \mathrm{Te}_{6}$ [1], $\mathrm{CrI}_{3}$ [2], $\mathrm{Fe}_{3} \mathrm{GeTe}_{2}$ $[3,4]$, and $\mathrm{VSe}_{2}$ [5], refreshes the conventional concept that at a finite temperature, ferromagnetic order in two-dimensional (2D) systems would collapse because of thermal fluctuation, according to the Mermin-Wagner theorem [6]. Unlike the conventional bulk ferromagnets in which 3D Heisenberg coupling dominates [7], the critical behavior in these vdW crystals may vary from one material to another. For example, bulk $\mathrm{CrI}_{3}$ and $\mathrm{Fe}_{3} \mathrm{GeTe}_{2}$ are consistent with 3D Heisenberg type behavior [8,9], but the Ising model is prevailing in $\mathrm{Cr}_{2} \mathrm{Si}_{2} \mathrm{Te}_{6}$ and $\mathrm{Cr}_{2} \mathrm{Ge}_{2} \mathrm{Te}_{6}[10,11]$ due to their different strengths of interlayer coupling. Moreover, even for the same material, the variation of thickness may lead to changes in magnetic properties. For example, bulk $\mathrm{CrI}_{3}$ presents 3D Heisenberg critical behavior [8], while a monolayer $\mathrm{CrI}_{3}$ is well described by the Ising model [2], unambiguously confirming the influence of the interlayer coupling. It is worth noting that in $\mathrm{Cr}_{2} \mathrm{Si}_{2} \mathrm{Te}_{6}$ and $\mathrm{Cr}_{2} \mathrm{Ge}_{2} \mathrm{Te}_{6}$, the magnetocrystalline anisotropy drives the systems to be $2 \mathrm{D}$ Ising ferromagnets, while the interlayer coupling is still non-negligible [12]. Thus, it is fundamentally interesting to draw a picture of magnetic critical behavior in such layered materials.

\footnotetext{
*These authors contributed equally to this work.

†Corresponding author: xixiang.zhang@kaust.edu.sa
}

The ternary $3 d$ transition-metal intercalates of niobium and tantalum dichalcogenides, $T_{x} M X_{2}(T=\mathrm{V}, \mathrm{Cr}, \mathrm{Mn}, \mathrm{Fe}, \mathrm{Co}$, $\mathrm{Ni} ; M=\mathrm{Nb}$, Ta; $X=\mathrm{S}$, Se; $x=1 / 4,1 / 3)$, are a quite large family of quasi-vdW magnetic materials that hold diverse magnetic orders depending on the chemical composition and crystal symmetry [13]. For example, $\mathrm{V}_{1 / 3} \mathrm{NbS}_{2}$ is a ferromagnet with an easy-plane anisotropy [13], while $\mathrm{Co}_{1 / 3} \mathrm{NbS}_{2}$ is antiferromagnetically ordered [14]. Additionally, $\mathrm{Cr}_{1 / 3} \mathrm{NbS}_{2}$ becomes a helimagnet that exhibits a chiral soliton lattice under a magnetic field applied in the $a b$ plane $[15,16]$. In particular, in $\mathrm{Cr}_{1 / 3} \mathrm{NbS}_{2}$, the formation of helimagnetic order confirms the strong coupling between neighboring layers, which is in good agreement with its 3D Heisenberg critical behavior $[17,18]$. It is noticeable that $\mathrm{Fe}_{x} \mathrm{TaS}_{2}$ (FTS) is the only member that holds ferromagnetic order accompanied with a strongly $c$-axis-aligned magnetic easy axis in this family [13], which is similar to those recently discovered 2D magnets [1-4]. It seems that in $\mathrm{Cr}_{2} \mathrm{Si}_{2} \mathrm{Te}_{6}$ and $\mathrm{Cr}_{2} \mathrm{Ge}_{2} \mathrm{Te}_{6}$, the $2 \mathrm{D}$ Ising model is valid probably due to the dominated magnetocrystalline anisotropy largely enshrouding the interlayer coupling. However, this has not been clearly verified in FTS. Moreover, the debate about the critical behavior of FTS has not been clarified even if different deductions have been drawn, i.e., FTS presents an Ising type spin state according to the X-ray absorption result [19], however the Heisenberg critical behavior is also observed in another study [20].

Here, we have successfully grown quasi-vdW singlecrystalline $\mathrm{Fe}_{0.26} \mathrm{TaS}_{2}$ samples and employed various techniques to determine the critical exponents so as to establish a picture of magnetism in $\mathrm{Fe}_{0.26} \mathrm{TaS}_{2}$. Critical exponents $\beta=$ $0.459(6)$ with a critical temperature $T_{C}=100.70(3) \mathrm{K}$ and 
$\gamma=1.205(11)$ with $T_{C}=100.67(3) \mathrm{K}$ are obtained by the Kouvel-Fisher (KF) method; whereas $\delta=3.69(1)$ is obtained by a critical isotherm analysis at $T_{C} \sim 100.7 \mathrm{~K}$. Renormalization group theory analysis reveals that the spin coupling in $\mathrm{Fe}_{0.26} \mathrm{TaS}_{2}$ is of the 3D Heisenberg ( $\{d: n=\{3: 3\})$ type with long-range magnetic interaction and that the exchange interaction decays with distance as $J(r) \sim r^{-(3+\sigma)}$ with $\sigma=1.71$.

\section{EXPERIMENTAL DETAILS}

High-quality bulk FTS single crystals were grown using the chemical vapor transport method [20]. First, pure elemental $\mathrm{Fe}, \mathrm{Ta}$, and $\mathrm{S}$ powders were mixed with a molar ratio of 0.4:1:2 and sealed in an evacuated quartz ampoule using $\mathrm{I}_{2}$ as a transport agent. During the growth process, the quartz ampoule was kept in a two-temperature-zone tube furnace for $7 \mathrm{~d}$, in which the temperatures of powder source and growth area were kept at 1000 and $900{ }^{\circ} \mathrm{C}$, respectively. Finally, millimeter-scale flakes with metallic luster were collected at the cooler end. The X-ray diffraction (XRD) data were acquired on a Bruker D2 PHASER diffractometer using $\mathrm{Cu} K_{\alpha}$ radiation $(\lambda=1.5418 \AA)$ at room temperature. The chemical characterization was implemented using energy-dispersive $\mathrm{x}$-ray spectroscopy in a Zeiss MERLIN scanning electron microscope. Rutherford backscattering spectrometry/channeling (RBS/C) measurements were performed using a $1.7 \mathrm{MeV} \mathrm{He}^{+}$ beam at the Ion Beam Center of Helmholtz-Zentrum DresdenRossendorf. The backscattered particles were detected at an angle of $170^{\circ}$ with respect to the incoming beam direction using silicon surface barrier detectors. Angular axial scans around different crystallographic directions were obtained using a two-axis goniometer. The magnetization measurements were performed with an MPMS3 magnetometer (Quantum Design). The external applied magnetic field $\left(H_{a}\right)$ was corrected to obtain the internal effective field $H$ as the existence of demagnetization effect: $H=H_{a}-N M$, where $M$ and $N$ are the measured magnetization and demagnetization factor, respectively, and $N$ was determined from the slope of the lowfield $M(H)$ curve following the method described in Ref. [21]. The corrected $H$ was then used for the scaling analysis.

\section{RESULTS AND DISCUSSION}

\section{A. Structure Characterization}

The crystal structure of $\mathrm{Fe}_{1 / 4} \mathrm{TaS}_{2}$ is illustrated in Fig. 1(a). Iron atoms are intercalated in the $\mathrm{vdW}$ gaps of $2 \mathrm{H}-\mathrm{TaS}_{2}$ slabs, and the whole crystal possesses a layered sandwich structure with a $2 a \times 2 a$ superlattice, where $a$ is the lattice constant of nonintercalated hexagonal $\mathrm{TaS}_{2}$. The as-grown single crystals are in plate-like form with metallic luster, as illustrated in Fig. 1(b). The actual elemental composition of the sample is confirmed by the energy-dispersive $\mathrm{x}$-ray spectrum (see Supplemental Material [22]), which is $\mathrm{Fe}_{0.26} \mathrm{TaS}_{2}$. In Fig. 1(c), the sharp $2 \theta$ XRD peaks, which can be indexed as the $(00 L)$ planes, demonstrate the excellent crystallinity of the sample. The $2 \theta$ XRD scan also indicates the slab surface of crystal is normal to the $c$ axis. Moreover, using Bragg's Law, we extracted the out-of-plane lattice parameter $c \approx 12.23 \AA$. In $2 \mathrm{H}-\mathrm{TaS}_{2}$, the density functional theory predicted and

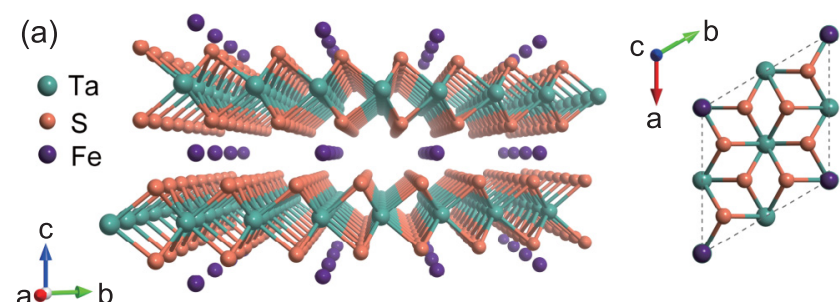

(b)

(c)
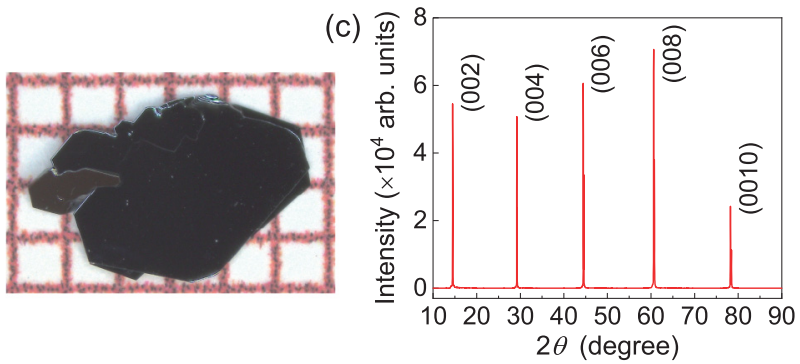

FIG. 1. (a) Schematic diagram of $\mathrm{Fe}_{1 / 4} \mathrm{TaS}_{2}$ crystal structure. Ta, $\mathrm{S}$, and $\mathrm{Fe}$ atoms are denoted by green, orange, and purple balls, respectively. The dashed lines in the top view indicate the crystal unit cell. (b) Optical image of a typical as-grown FTS crystal. The size of the grid is $1 \mathrm{~mm}^{2}$. (c) XRD pattern of FTS measured with the slab surface lying flat on the sample holder.

experimental $c$-axis lattice parameter are $12.54 \AA$ [23] and $12.07 \AA$ [24], respectively. The difference in lattice constant between $2 \mathrm{H}-\mathrm{TaS}_{2}$ and our sample probably be caused by the intercalation of iron atoms. Besides, our result is between the values of $12.154 \AA$ for $\mathrm{Fe}_{1 / 4} \mathrm{TaS}_{2}$ and $12.284 \AA$ for $\mathrm{Fe}_{1 / 3} \mathrm{TaS}_{2}[20,25]$.

To further reveal the occupation of $\mathrm{Fe}$ in the material and the crystallization of the sample, we performed the RBS/C spectroscopy and angular-scan mapping around three major crystalline axes. The red curve in Fig. 2(a) is the fitted RBS random spectra for $\mathrm{Fe}_{0.26} \mathrm{TaS}_{2}$ using the SIMNRA code $[26,27]$, which is well consistent with the experimental signals. For the RBS/C measurement, the $\mathrm{He}^{+}$beam is aligned along the $c$ axis of the sample for the channel exploration, and the sharply reduced yield backscattering signal indicates a highly ordered period potential along the aligned crystal $c$ axis, undoubtedly confirming the single crystalline nature of the sample. To further investigate the $\mathrm{Fe}$ distribution, a two-dimensional backscattered-yield mapping measurement from Fe RBS/C was performed, and the result is displayed in Fig. 2(b). As expected, by changing the incident angle of $\mathrm{He}^{+}$, the channels in the vicinity of the $\langle 311\rangle,\langle 111\rangle$, and $\langle 110\rangle$ directions are observed, which indicates that the $\mathrm{Fe}$ atoms are located at the ordered positions rather than being intercalated randomly. Therefore, as confirmed by the results from XRD and RBS/C data, the excellent single-crystal sample was used in our study.

\section{B. Magnetic Properties}

To systematically investigate the magnetic properties of FTS, magnetic moments as a function of effective magnetic field and temperature-dependent magnetization were measured in a wide range of temperatures and magnetic fields. 


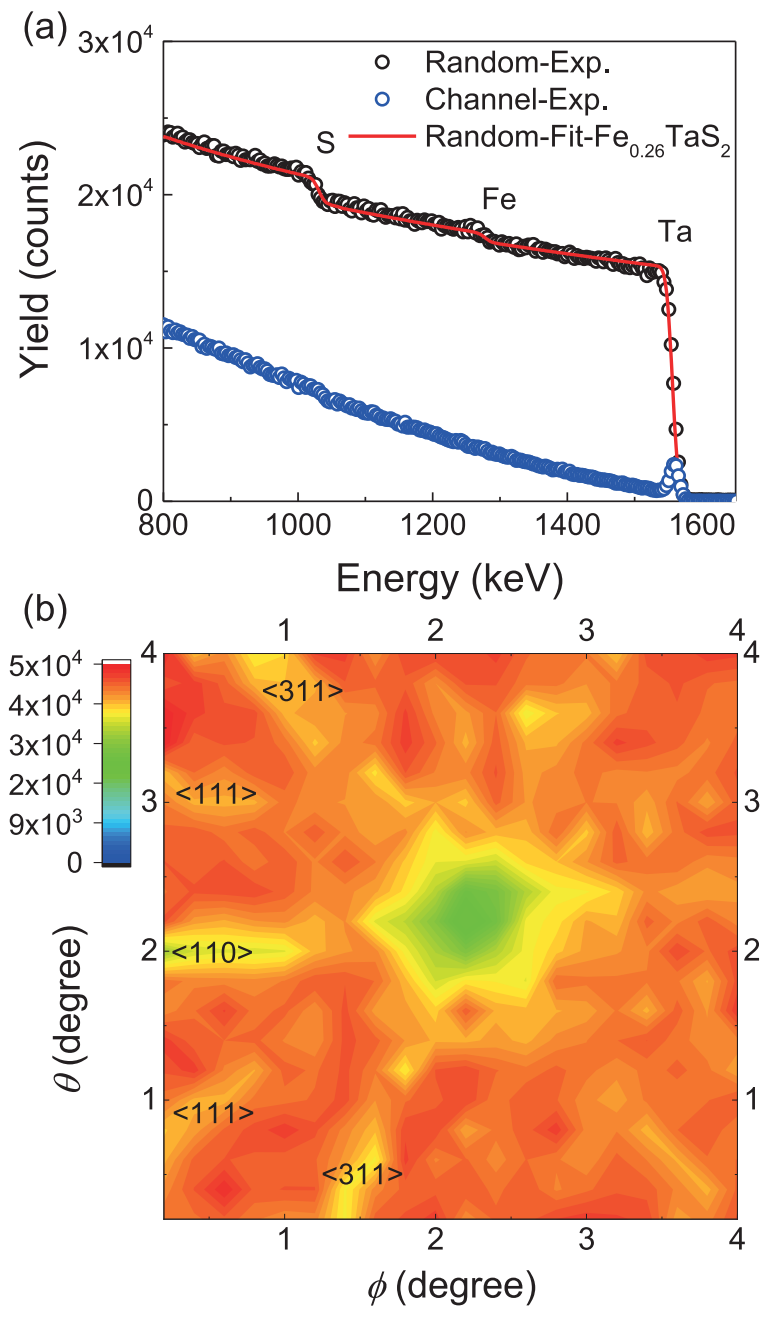

FIG. 2. (a) Experimental random (black) and channeling (blue) RBS spectra of the single-crystalline $\mathrm{Fe}_{0.26} \mathrm{TaS}_{2}$ sample with the $\mathrm{He}^{+}$ beam aligned along $c$ axis. The red line is the fitted RBS random spectra for $\mathrm{Fe}_{0.26} \mathrm{TaS}_{2}$ using the SIMNRA code. (b) Experimental two-dimensional backscattered-yield mapping patterns of the $\mathrm{Fe}$ in the crystal obtained from RBS/C spectra. $\theta$ and $\phi$ are the scanning angles when performing the two-dimensional mapping, and the color scale is the integral backscattering signals (counts).

Some representative data are presented in Fig. 3. Square hysteresis loops were observed when the field was applied along the $c$ axis at low temperatures. Furthermore, a very large coercivity up to $\sim 60 \mathrm{kOe}$ was observed at $6 \mathrm{~K}$ in the $H \| c$ configuration. However, when the field was applied in the $a b$ plane, only a linear dependent $M(H)$ curve could be obtained even with the field up to $70 \mathrm{kOe}$ at $6 \mathrm{~K}$ with much smaller magnetic moments than measured for $H \| c$. These results unambiguously suggest that this huge magnetocrystalline anisotropy should be much stronger than shape anisotropy (or the demagnetization effect) and that $\mathrm{Fe}_{0.26} \mathrm{TaS}_{2}$ single crystal has a uniaxial anisotropy along the $c$ axis. It is worth noting that the measured saturation moment of $\mathrm{Fe}$ ions is about $3.96 \mu_{B}$, corresponding to the high-spin state of $\mathrm{Fe}^{2+}\left(4 \mu_{B}\right)$. Figure 3(b) presents the temperature-dependent magnetization $M(T)$ curves measured at a 100 Oe magnetic
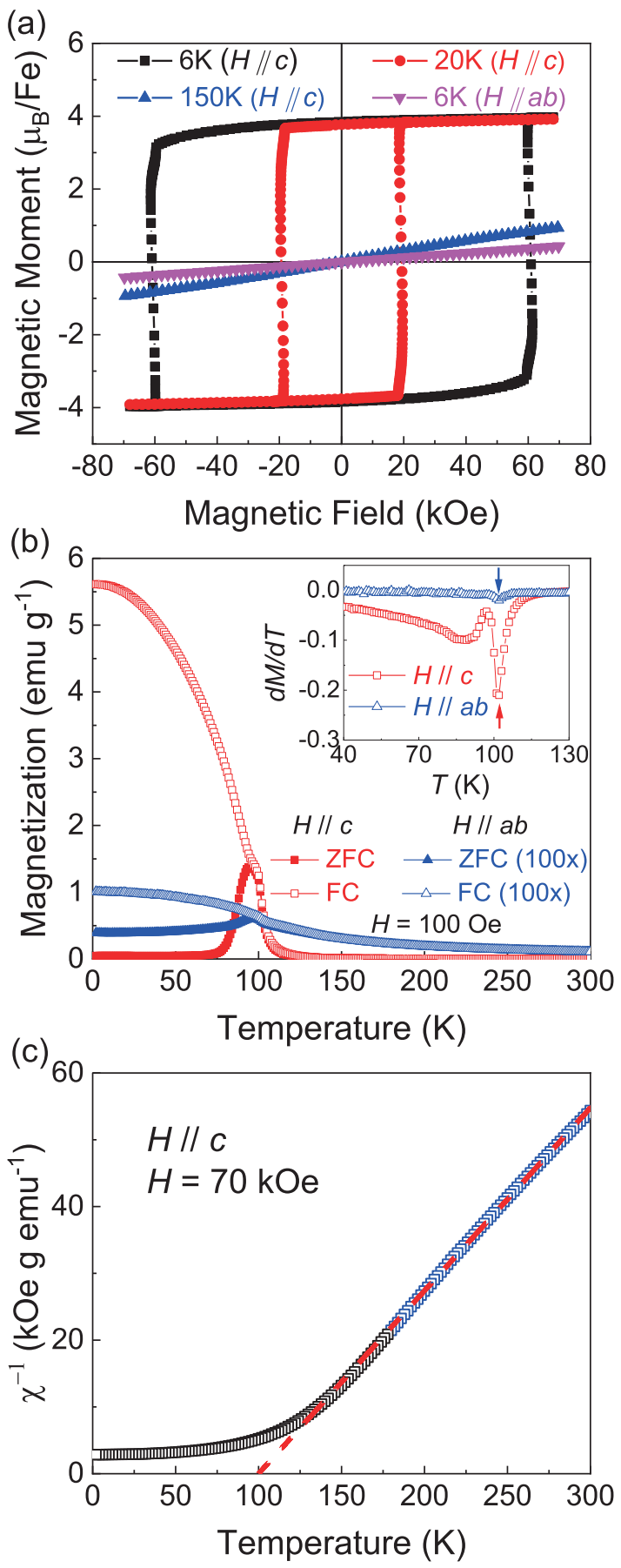

FIG. 3. (a) Magnetic-field-dependent magnetic moment of a $\mathrm{Fe}_{0.26} \mathrm{TaS}_{2}$ sample measured at different temperatures with the field being applied along the $c$ axis and in the $a b$ plane, respectively. (b) Temperature-dependent magnetization curves after zero-fieldcooling (solid) and field-cooling (open) processes followed by a 100 Oe magnetic field being applied along the $c$ axis (red squares) and in the $a b$ plane (blue triangles), respectively. The lines for the $H \| a b$ plane case are enlarged 100 times for clarity. Inset: the $d M / d T$ vs $T$ of field-cooling $M(T)$ curves. (c) Temperature dependence of inverse susceptibility with a $70 \mathrm{kOe}$ field applied along the $c$ axis. The red dashed line describes the fitting from the Curie-Weiss equation in which the temperature region is chosen from 180 to $300 \mathrm{~K}$ as highlighted in blue. 
field applied along the $c$ axis and in the $a b$ plane, respectively, after zero-field-cooling (ZFC) and field-cooling (FC) sequences. One can observe apparent ferromagnetic (FM) to paramagnetic (PM) transitions from both field configurations when the temperature increases. The first derivative of FC curves is shown in the inset in Fig. 3(b). For both $H \| c$ and $H \| a b$ cases, a same transition temperature of $102 \mathrm{~K}$ is determined roughly from the minimal point of the $d M / d T$ curves, which is pointed out by arrows for clarity. In the $M(T)$ curves for $H \| c$ configuration, we also observe an abrupt increase of magnetization below $T_{C}$. This phenomenon was also observed by other researchers and it was ascribed to the spin fluctuations near $T_{C}$ [20]. To obtain more information about the mechanism of the magnetism in this material, we fit the data of the magnetization as a function of temperature obtained in the temperature range of 180-300 K (much higher than the transition temperature of $\sim 102 \mathrm{~K}$ ) under a magnetic field of $70 \mathrm{kOe}$ with $H \| c$ axis to the Curie-Weiss law $\chi=$ $\frac{C}{T-\theta}$, where $C$ and $\theta$ are the Curie-Weiss constant and Weiss temperature, respectively. The fitting data are presented in Fig. 3(c), in which a good linear fitting leads to $\theta \approx 100 \mathrm{~K}$. The positive Weiss temperature indicates the apparent ferromagnetic interaction in FTS, and the value is close to the one deduced from $d M / d T$ curves. Simultaneously, we also extracted the effective magnetic moment $\mu_{\text {eff }}=5.39 \mu_{B}$ using the relation $C=N_{A} \mu_{B}^{2} \mu_{\text {eff }}^{2} /\left(3 k_{B}\right)$ [28], which is close to a previous report [20]. We note that the effective magnetic moment is considerably larger than the spin-only moment of $\mathrm{Fe}^{2+}$ of $4.9 \mu_{B}$. That means there is a nonquenched orbital angular momentum contribution to $\mathrm{Fe}^{2+}$ in $\mathrm{Fe}_{0.26} \mathrm{TaS}_{2}$, which has been demonstrated by other studies $[13,19,29]$. In addition, we calculated the Rhodes-Wohlfarth ratio $q_{c} / q_{s}[30,31]$, where $q_{c}$ is obtained from $q_{c}\left(q_{c}+2\right)=\mu_{\text {eff }}^{2}$ and $q_{s}=3.96 \mu_{B}$ is the saturation magnetization directly measured at a low temperature $(6 \mathrm{~K})$. In this scenario, a metallic ferromagnet with $q_{c} / q_{s} \sim 1$ can be treated as a localized system, while the itinerant system has $q_{c} / q_{s}>1$. For FTS, we obtained $q_{c} / q_{s}=1.13$, which indicates the weak itinerant character in FTS. In fact, it was found that the iron concentration $x$ in $\mathrm{Fe}_{x} \mathrm{TaS}_{2}$ can be tuned from 0.15 to 0.50 during the growth process $[29,32,33]$. The ferromagnetic coupling in $\mathrm{Fe}_{x} \mathrm{TaS}_{2}$ is considered as the Ruderman-Kittel-Kasuya-Yosida interaction in which the local spins of intercalated $\mathrm{Fe}$ ions align ferromagnetically through the itinerant Ta $5 d$ electrons, and this indirect exchange interaction is maximized when the Fe ions locate at the $2 a \times 2 a$ superlattice sites, which makes $\mathrm{Fe}_{1 / 4} \mathrm{TaS}_{2}$ reach a maximum Curie temperature of $\sim 160 \mathrm{~K}$ [19]. As the $\mathrm{Fe}$ concentration in our samples is $x \approx 0.26$, the transition temperature we obtain is much lower than $\mathrm{Fe}_{1 / 4} \mathrm{TaS}_{2}$. But this concentration, $x \approx 0.26$, should bring FTS a maximum coercivity [33].

\section{Critical Behavior}

To determine the Curie temperature more precisely and gain insight into the magnetic transition nature in FTS, we measured the isothermals in the temperature range of 96-106 K with the $H \| c$ configuration, as shown in Fig. 4(a). The data in Fig. 4(a) were then replotted in the form of the
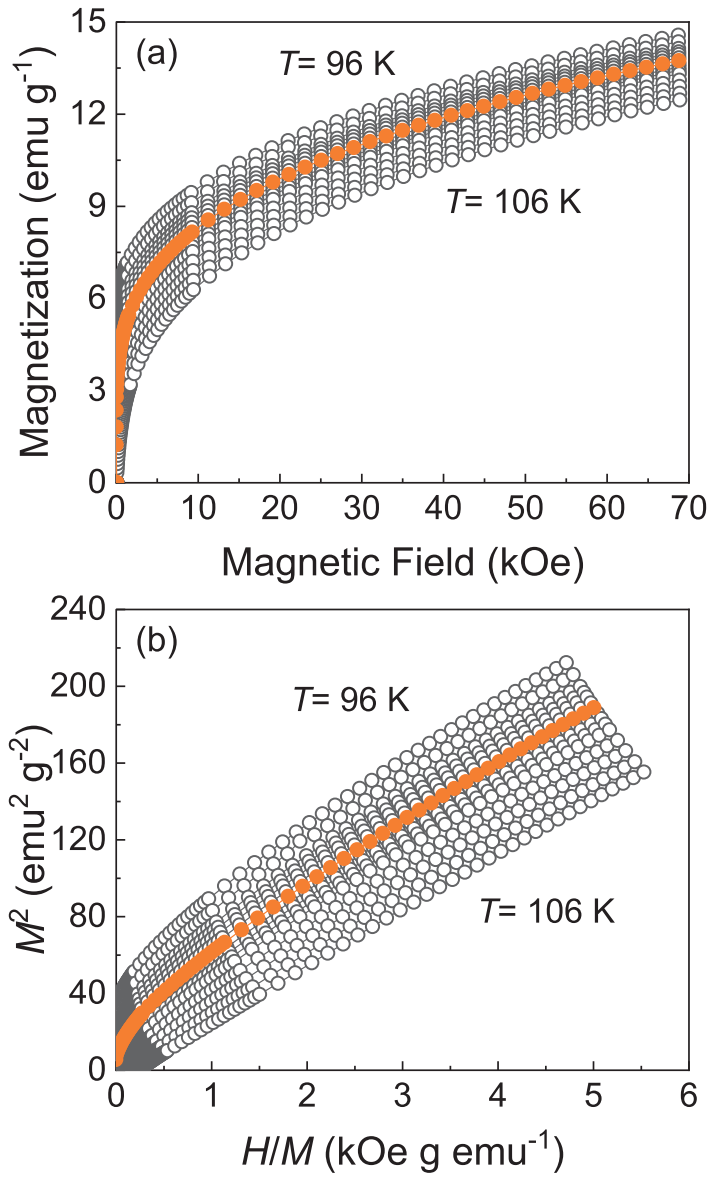

FIG. 4. (a) Magnetic-field-dependent initial isothermal magnetization curves measured from 96 to $106 \mathrm{~K}$ with $H \| c$, and (b) is the corresponding Arrott plot. The orange-highlighted solid curve represents the measurement taking place at the Weiss temperature of $100 \mathrm{~K}$ estimated by the Curie-Weiss law. In the range of 98-102 K, the temperature step is $0.5 \mathrm{~K}$. Otherwise, it is $1 \mathrm{~K}$.

Arrott plot [34], that is, $M^{2}$ vs $H / M$ as shown in Fig. 4(b). In the description of the Arrott plot, assuming critical behavior obeys the Landau mean-field theory, the $M^{2}$ vs $H / M$ curves should be straight and parallel to each other in the highfield region. Additionally, the temperature at which the curve passes through the origin point can be determined as the critical temperature $T_{C}$. In Fig. 4(b), one can see that all the curves are not straight or parallel even in the high-field region, which suggests that the ideal mean-field theory cannot describe the critical behavior perfectly in FTS. This is not surprising because Coulomb correlation and spin fluctuations are neglected in the mean-field theory, while they can be significant in an itinerant ferromagnet [35]. Moreover, these curves measured around the phase transition temperature provide other information. According to the Banerjee criterion [7], one can distinguish first-order magnetic transitions from second-order ones by the signs of the slopes of $M^{2}$ vs $H / M$ curves around the transition temperature. That is, negative slopes correspond to first-order transitions while positive slopes correspond to second-order transitions. Therefore, the Arrott plot in Fig. 4(b) confirms a second-order FM-PM transition in FTS. 

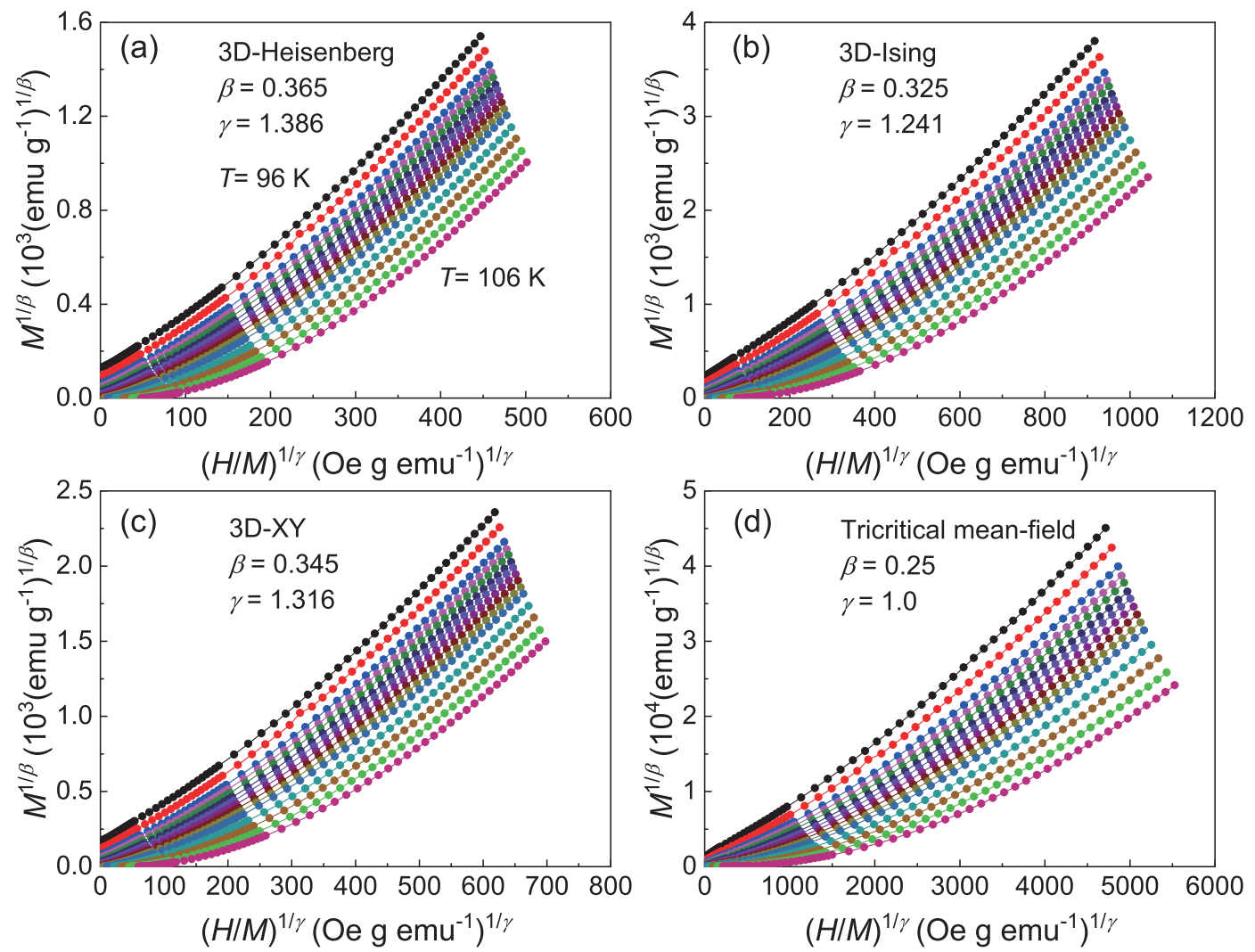

FIG. 5. The modified Arrott plots of $M^{1 / \beta}$ vs $(H / M)^{1 / \gamma}$ in the description of (a) 3D Heisenberg model $(\beta=0.365, \gamma=1.386)$, (b) 3D Ising model $(\beta=0.325, \gamma=1.241)$, (c) 3D XY model $(\beta=0.345, \gamma=1.316)$, and (d) tricritical mean-field model $(\beta=0.25, \gamma=1.0)$.

Generally, the critical behavior of a second-order phase transition can be depicted by a series of interrelated critical exponents. Since the standard Arrott plot cannot describe the magnetic interactions properly in FTS, the critical magnetization isotherms were then reanalyzed with the Arrott-Noakes equation of state [36]:

$$
(H / M)^{1 / \gamma}=a \varepsilon+b M^{1 / \beta},
$$

where $\varepsilon=\left(T-T_{C}\right) / T_{C}$ is the reduced temperature, $a$ and $b$ are material constants, and $\beta$ and $\gamma$ are critical exponents that are respectively associated with the spontaneous magnetization $M_{S}(T)$ below $T_{C}$ and the inverse initial susceptibility $\chi_{0}^{-1}(T)$ above $T_{C}$ by the following power laws [37]:

$$
\begin{gathered}
M_{S}(T)=M_{0}(-\varepsilon)^{\beta}, \quad T<T_{C}, \quad \varepsilon<0, \\
\chi_{0}^{-1}(T)=\left(h_{0} / m_{0}\right) \varepsilon^{\gamma}, \quad T>T_{C}, \quad \varepsilon>0, \\
M=D H^{1 / \delta}, \quad T=T_{C}, \quad \varepsilon=0,
\end{gathered}
$$

where $\delta$ is another critical exponent associated with the magnetization isotherm at $T_{C}$, and $M_{0}, h_{0} / m_{0}$, and $D$ are the critical amplitudes. In the asymptotic second-order magnetic phase transition critical region, appropriate values of $\beta, \gamma$, and $\delta$ should give rise to a set of parallel straight lines of $M^{1 / \beta}$ vs $(H / M)^{1 / \gamma}$ in the high-field region. At $T=T_{C}$, the line should pass through the origin. Additionally, the linear extrapolation of the high-field straight line should intercept the $M^{1 / \beta}$ axis, giving the value of $M_{S}(T)$ below $T_{C}$, and intercept the $(H / M)^{1 / \gamma}$ axis, giving the value of $\chi_{0}^{-1}(T)$ above $T_{C}$, respectively. Typically, there are four universal models: the 3D Heisenberg model $(\beta=0.365, \gamma=1.386)$ [38], 3D Ising model $(\beta=0.325, \gamma=1.241)$ [38], 3D XY model $(\beta=0.345, \gamma=1.316)$ [39], and tricritical mean-field model ( $\beta=0.25, \gamma=1.0$ ) [40]. These models, presented in Fig. 5, are usually used to establish the relation between $M^{1 / \beta}$ and $(H / M)^{1 / \gamma}$. However, all these modified Arrott plots do not yield parallel straight lines, indicating these four models are not valid in FTS or the magnetic interaction in FTS cannot be described solely by one single model.

For purpose of achieving precise critical exponents as well as critical temperature, a rigorous iterative method has been adopted [21]. In the modified Arrott plot, the linear extrapolations of the curves in the high-field region intercepting the $M^{1 / \beta}$ and $(H / M)^{1 / \gamma}$ axes yield reliable values of $M_{S}(T)$ and $\chi_{0}^{-1}(T)$, respectively. These obtained $M_{S}(T)$ and $\chi_{0}^{-1}(T)$ values can be utilized to fit $\beta$ and $\gamma$ by following Eqs. (2) and (3). Then, the fitted exponents are used to construct a modified Arrott plot. This exercise is repeated until both $\beta$ and $\gamma$ are convergent. It should be noted that the exponents and critical temperature obtained by this method are not sensitive to the initial parameters [10]. The final converged critical exponents $\beta=0.460$ (4), with $T_{C}=100.68(1) \mathrm{K}$, and $\gamma=1.216(11)$, with $T_{C}=100.65(2) \mathrm{K}$, are presented in Fig. 6(a). Moreover, Figure 6(b) illustrates the corresponding modified Arrott plot, in which one can see that all the isotherms in the high-field region combine a set of parallel straight lines, verifying that the obtained critical exponents are reliable. 

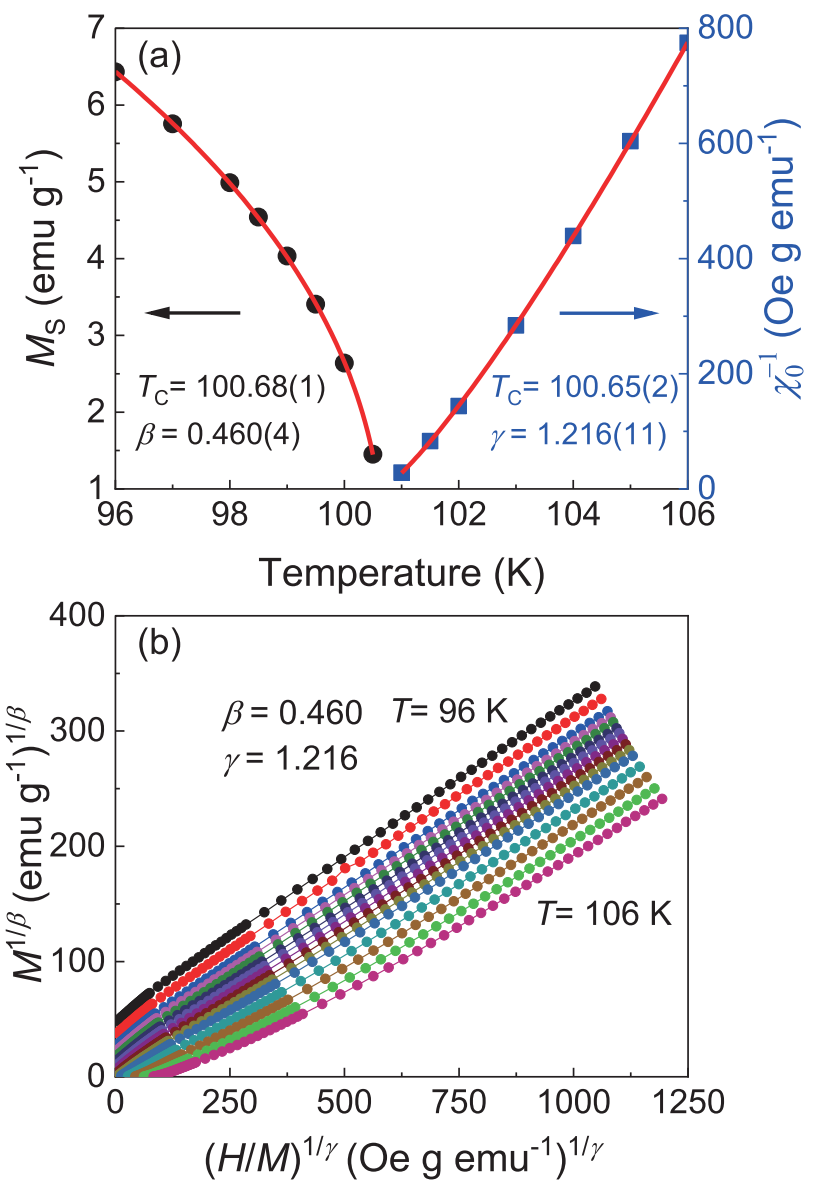

FIG. 6. (a) Temperature-dependent spontaneous magnetization $M_{S}$ (left) and inverse initial susceptibility $\chi_{0}^{-1}$ (right) with red fitting curves for FTS. (b) The modified Arrott plot of $M^{1 / \beta}$ vs $(H / M)^{1 / \gamma}$ with parameters of $\beta=0.460$ and $\gamma=1.216$.

Simultaneously, we have also employed the KF method to determine the critical exponents as well as critical temperature [41]:

$$
\begin{gathered}
\frac{M_{S}(T)}{d M_{S}(T) / d T}=\frac{T-T_{C}}{\beta}, \\
\frac{\chi_{0}^{-1}(T)}{d \chi_{0}^{-1}(T) / d T}=\frac{T-T_{C}}{\gamma} .
\end{gathered}
$$

In this way, $M_{S}(T) /\left[d M_{S}(T) d T\right]$ and $\chi_{0}^{-1}(T) /\left[d \chi_{0}^{-1}(T) /\right.$ $d T]$ should show linear dependences on temperature with slopes of $\beta / 1$ and $\gamma / 1$, respectively, and the intercepts of fitted straight lines on the temperature axis should yield the critical temperature. Figure 7(a) illustrates the KF plot for our sample, in which the linear functions in the region below and above $T_{C}$ give $\beta=0.459(6)$, with $T_{C}=$ 100.70(3) K, and $\gamma=1.205(11)$, with $T_{C}=100.67(3) \mathrm{K}$, respectively. The values of critical exponents and critical temperature from the KF plots are consistent with those obtained from the iterative modified Arrott plot, which confirms that the estimated parameters are self-consistent and intrinsic.
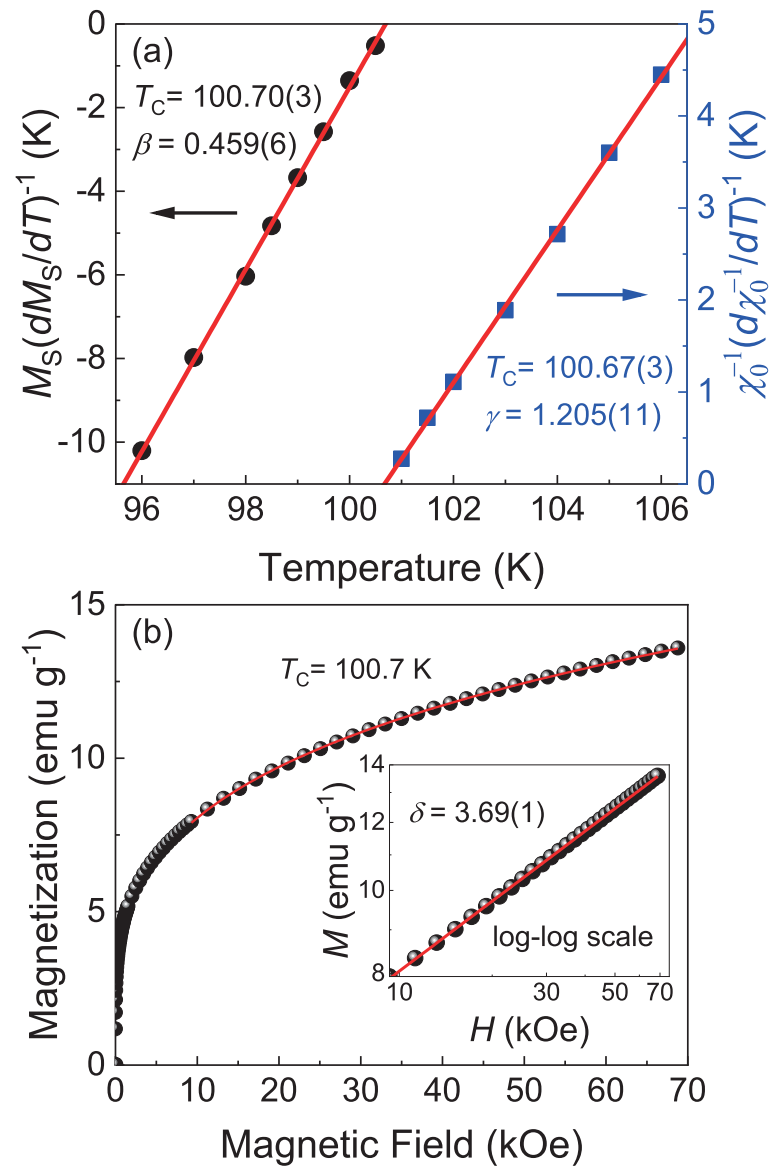

FIG. 7. (a) The $\mathrm{KF}$ plot of $M_{S}(T) /\left[d M_{S}(T) d T\right]$ (left) $\chi_{0}^{-1}(T) /\left[d \chi_{0}^{-1}(T) / d T\right]$ and (right) with red fitting curves for FTS. (b) The isotherm magnetic-field-dependent magnetization measured at $T_{C}=100.7 \mathrm{~K}$ with a red fitting curve. Inset: the corresponding log-log scale plot.

Another critical exponent $\delta$ can be achieved according to the Widom scaling relation [42]:

$$
\delta=1+\frac{\gamma}{\beta} .
$$

Accordingly, we obtained $\delta=3.64(3)$ and $\delta=3.63(4)$ based on the $\beta$ and $\gamma$ values coming from modified Arrott plot and KF method, respectively. Alternatively, $\delta$ can also be determined directly from the inverse slope of the critical isotherm in logarithmic scale according to Eq. (4). Figure 7(b) presents the isothermal magnetization $M(H)$ measured at critical temperature $T_{C} \sim 100.7 \mathrm{~K}$ deduced by the modified Arrott plot and KF method. A linear fit of $\lg (M)$ vs $\lg (H)$ curve at the high-field range $(H>10 \mathrm{kOe})$ results in $\delta=3.69(1)$, as illustrated in the inset of Fig. 7(b). The critical isothermal analysis is in agreement with the result from the Widom scaling law. Therefore, all the critical exponents $\beta$, $\gamma, \delta$, and $T_{C}$ obtained in our study are self-consistent and reasonably accurate within experimental precision.

The reliability of the obtained critical exponents and critical temperature has been verified by scaling analysis. For magnetic systems in their critical asymptotic region, the 

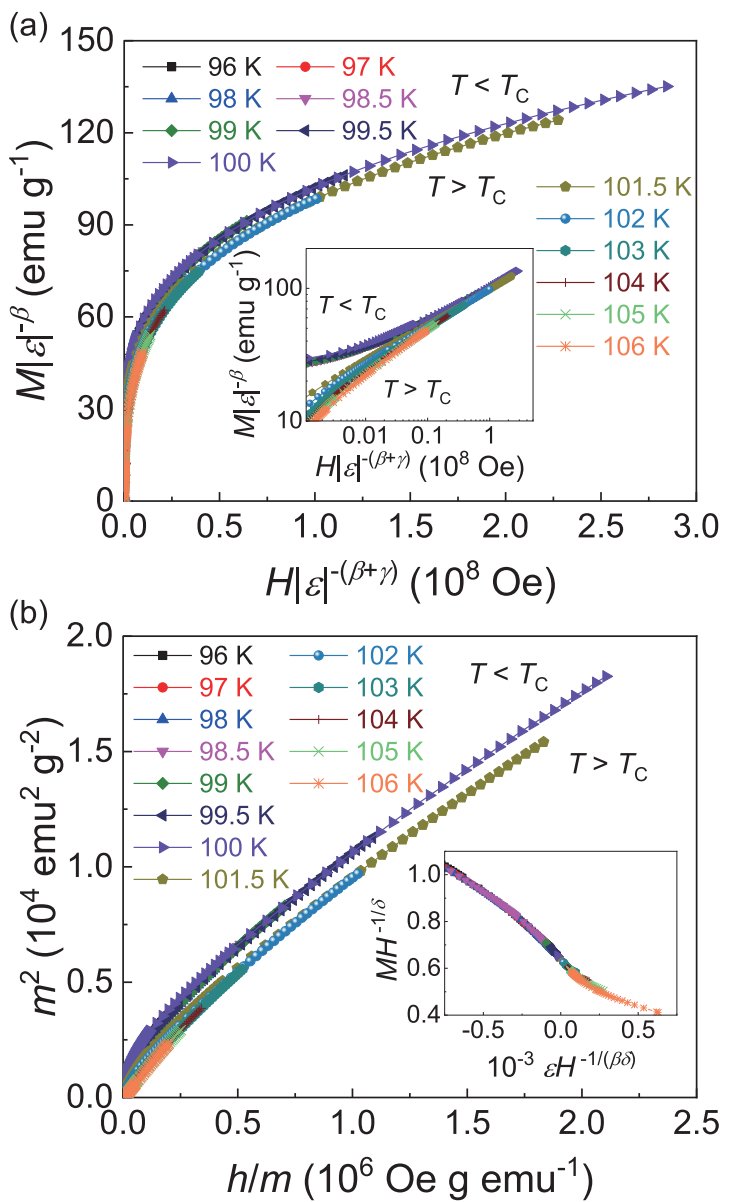

FIG. 8. (a) Renormalized magnetization $m$ as a function of renormalized magnetic field $h$ below and above $T_{C}$ for FTS. Inset: the corresponding log-log scale plot. (b) The $m^{2}$ vs $h / m$ plot. Inset: the plot of $M H^{-1 / \delta}$ vs $\varepsilon H^{-1 /(\beta \delta)}$.

scaling equation of state can be expressed as [43]

$$
M(H, \varepsilon)=\varepsilon^{\beta} f_{ \pm}\left(H / \varepsilon^{\beta+\gamma}\right),
$$

where $f_{ \pm}$are the regular functions defined as $f_{+}$for $\varepsilon>0$ and $f_{-}$for $\varepsilon<0$, respectively. Equation (8) can also be written as

$$
m=f_{ \pm}(h),
$$

where $m \equiv \varepsilon^{-\beta} M(H, \varepsilon)$ is the renormalized magnetization and $h \equiv \varepsilon^{-(\beta+\gamma)} H$ is the renormalized field, respectively. Such a scaling hypothesis implies that if the $\beta, \gamma$, and $\delta$ values are appropriately chosen, the scaled $m$ and $h$ should fall on two universal curves: one for $\varepsilon>0$ and the other for $\varepsilon<0$. According to Eq. (9), the scaled $m$ vs $h$ curves are plotted in Fig. 8(a). Obviously the lines are separated into two branches, which is more visible in the logarithmic scale shown in the inset of Fig. 8(a). This can be further verified by a more rigorous method using an $m^{2}$ vs $h / m$ plot [38], as shown in Fig. 8(b), in which all the curves also collapse into two divided branches. Moreover, the scaling equation of state has another form [43]

$$
\frac{H}{M^{\delta}}=k\left(\frac{\varepsilon}{H^{1 / \beta}}\right),
$$

where $k(x)$ is the scaling function. According to Eqs. (5) and (10), the plot of $M H^{-1 / \delta}$ vs $\varepsilon H^{-1 /(\beta \delta)}$ should correspond to one universal curve [44]. As expected, all the data fall on one single curve, as shown in the inset in Fig. 8(b). The well-rescaled curves further ensure that the obtained critical exponents and $T_{C}$ are reliable and accordant with the scaling hypothesis.

The critical exponents for FTS obtained using different methods as well as different theoretical models are summarized in Table I. Some other layered magnets are also listed for comparison. It seems that the experimentally obtained exponents of FTS cannot be categorized into any conventional universality class. The exponent $\beta$ lies between mean-field model and 3D Heisenberg model but quite closer to the former, which suggests the exchange interaction in FTS is likely to be a long-range type. However, $\gamma$ is near 3D Ising model, which could be attributed to the apparent out-of-plane magnetic anisotropy in FTS. A comprehensive investigation has demonstrated that the critical exponent $\beta$ for a 2D magnet should be located in a universal window of $\sim 0.1 \leqslant \beta \leqslant$ 0.25 [45]. As expected, $\mathrm{Cr}_{2} \mathrm{Si}_{2} \mathrm{Te}_{6}$ and $\mathrm{Cr}_{2} \mathrm{Ge}_{2} \mathrm{Te}_{6}$ have $\beta$ values inside this window and they both demonstrate $2 \mathrm{D}$ Ising type coupling [10,11]. While $\mathrm{CrI}_{3}, \mathrm{Fe}_{3} \mathrm{GeTe}_{2}$, and $\mathrm{Mn}_{3} \mathrm{Si}_{2} \mathrm{Te}_{6}$ are all found to exhibit the 3D critical phenomenon with $\beta>0.25[8,9,46-48]$. It is interesting that $3 \mathrm{D}$ magnetic characteristics are observed in vdW-bonded magnets, and it could be explained by the fact that $\mathrm{CrI}_{3}$ and $\mathrm{Fe}_{3} \mathrm{GeTe}_{2}$ have smaller vdW gaps than $\mathrm{Cr}_{2} \mathrm{Si}_{2} \mathrm{Te}_{6}$ and $\mathrm{Cr}_{2} \mathrm{Ge}_{2} \mathrm{Te}_{6}$ [46,49], which gives rise to much stronger interlayer coupling. It is also worth mentioning that $\mathrm{Mn}_{3} \mathrm{Si}_{2} \mathrm{Te}_{6}$ has a similar crystal structure with FTS and that $\mathrm{Mn}_{2} \mathrm{Si}_{2} \mathrm{Te}_{6}$ layers are connected by another $\mathrm{Mn}$ atom located inside the interlayer space. Such a structure promises $\mathrm{Mn}_{3} \mathrm{Si}_{2} \mathrm{Te}_{6}$ robust interlayer coupling as well as a 3D long-range magnetic interaction [48]. We can also see that FTS has critical exponents quite close to $\mathrm{Mn}_{3} \mathrm{Si}_{2} \mathrm{Te}_{6}$, so it is reasonable to expect FTS to show similar critical behavior as observed in $\mathrm{Mn}_{3} \mathrm{Si}_{2} \mathrm{Te}_{6}$.

It is known that the universality class of the magnetic phase transition depends on the range of the exchange interaction $J(r)$. According to renormalization group theory analysis, the interaction decays with distance $r$ as $J(r) \sim e^{-r / b}$ for shortrange exchange and $J(r) \sim r^{-(d+\sigma)}$ for long-range exchange, respectively, where $r$ is the distance, $b$ is the spatial scaling factor, $d$ is the dimension of the system, and $\sigma$ is the range of exchange interaction [51,52]. The relationship between $\sigma$ and critical exponent $\gamma$ can be expressed as follows [51]:

$$
\begin{aligned}
\gamma= & +\frac{4}{d}\left(\frac{n+2}{n+8}\right) \Delta \sigma \\
& +\frac{8(n+2)(n-4)}{d^{2}(n+8)^{2}}\left[1+\frac{2 G\left(\frac{d}{2}\right)(7 n+20)}{(n-4)(n+8)}\right] \Delta \sigma^{2},
\end{aligned}
$$

where $\Delta \sigma=\sigma-\frac{d}{2}, G\left(\frac{d}{2}\right)=3-\frac{1}{4}\left(\frac{d}{2}\right)^{2}$ and $n$ is the spin dimensionality. The renormalization group theory analysis also suggests that if $\sigma>2$, it indicates a short-range interaction in the system, while $\sigma<2$ implies a long-range type of interaction, and the mean-field model ( $\beta=0.5, \gamma=1.0$, and $\delta=3.0)$ should satisfy when $\sigma<\frac{d}{2}[51,52]$. To better understand the exchange interaction in FTS, we have followed 
TABLE I. Comparison of critical exponents of $\mathrm{Fe}_{0.26} \mathrm{TaS}_{2}$ and some other layered magnets derived by various methods.

\begin{tabular}{|c|c|c|c|c|c|c|c|}
\hline Material & Reference & Technique & $\beta$ & $\gamma$ & $\delta$ & $\{d: n\}$ & $J(r)$ \\
\hline \multirow[t]{8}{*}{$\mathrm{Fe}_{0.26} \mathrm{TaS}_{2}$} & This work & Iterative modified Arrott plot & $0.460(4)$ & $1.216(11)$ & $3.64(3)$ & \multirow[t]{8}{*}{$3: 3$} & \multirow[t]{8}{*}{$r^{-4.71}$} \\
\hline & This work & KF method & $0.459(6)$ & $1.205(11)$ & $3.63(4)$ & & \\
\hline & This work & Critical isotherm & & & $3.69(1)$ & & \\
\hline & [38] & Mean-field (theory) & 0.5 & 1.0 & 3 & & \\
\hline & [38] & 3D Heisenberg (theory) & 0.365 & 1.386 & 4.80 & & \\
\hline & [38] & 3D Ising (theory) & 0.325 & 1.241 & 4.82 & & \\
\hline & [39] & 3D XY (theory) & 0.345 & 1.316 & 4.81 & & \\
\hline & {$[40]$} & Tricritical mean-field (theory) & 0.25 & 1.0 & 5 & & \\
\hline $\mathrm{Cr}_{2} \mathrm{Si}_{2} \mathrm{Te}_{6}$ & [10] & KF method & $0.175(9)$ & $1.562(9)$ & $9.925(56)$ & $\{2: 1\}$ & $r^{-3.63}$ \\
\hline \multirow[t]{2}{*}{$\mathrm{Cr}_{2} \mathrm{Ge}_{2} \mathrm{Te}_{6}$} & [11] & KF method & $0.200(3)$ & $1.28(3)$ & $7.40(5)$ & \multirow[t]{3}{*}{$2: 1$} & $r^{-3.52}$ \\
\hline & {$[50]$} & KF method & $0.240(6)$ & $1.000(5)$ & $5.167(5)$ & & \\
\hline $\mathrm{CrI}_{3}$ & [8] & KF method & $0.260(4)$ & $1.136(6)$ & $5.37(4)$ & & $r^{-4.69}$ \\
\hline $\mathrm{Fe}_{2.64(6)} \mathrm{Ge}_{0.87(4)} \mathrm{Te}_{2}$ & [9] & KF method & $0.372(4)$ & $1.265(15)$ & $4.401(6)$ & \multirow[t]{4}{*}{$3: 3$} & $r^{-4.89}$ \\
\hline $\mathrm{Fe}_{3} \mathrm{GeTe}_{2}$ & [46] & KF method & $0.322(4)$ & $1.063(8)$ & $4.301(65)$ & & $r^{-4.6}$ \\
\hline $\mathrm{Fe}_{2.85} \mathrm{GeTe}_{2}$ & [47] & KF method & 0.363 & 1.228 & 4.398 & & $r^{-4.8}$ \\
\hline $\mathrm{Mn}_{3} \mathrm{Si}_{2} \mathrm{Te}_{6}$ & [48] & KF method & $0.41(1)$ & $1.21(2)$ & $3.95(2)$ & & $r^{-4.79}$ \\
\hline
\end{tabular}

the procedure similar to that employed in Ref. [52] to obtain the value of $\sigma$. That is, $\sigma$ is chosen for a particular set of $\{d: n\}$ that yields a value of $\gamma$ close to the experimental value $(\sim 1.2)$ using Eq. (11). The other exponents can be calculated from the following scaling relations: $v=\gamma / \sigma, \alpha=2-v d$, $\beta=(2-\alpha-\gamma)$, and $\delta=1+\gamma / \beta$. The process is repeated for different sets of $\{d: n\}$, and we found $\{d: n\}=\{3: 3\}$ and $\sigma=1.71$ give exponents $(\beta=0.436, \gamma=1.155$, and $\delta=3.65$ ) that match well with the experimental values (see Table I). Such a result indicates the spin interaction in FTS is of the $3 \mathrm{D}$ Heisenberg $(\{d: n\}=\{3: 3\})$ type with longrange magnetic coupling and that the exchange decays with distance as $J(r) \sim r^{-4.71}$. This conclusion is consistent with our previous analysis.

Long-range interaction and magnetocrystalline anisotropy are two strategies for a magnet to be exempt from the MerminWagner theorem [53]. The bulk 3D ferromagnet FTS evidently owns these two features. The weak interlayer bonding of FTS implies the possibility of achieving its 2D sheets through mechanical exfoliation. In practice, we have demonstrated that 2D FTS crystals are indeed procurable through microexfoliation (see Supplemental Material [22]). Therefore, it is of great interest to explore the magnetic properties of FTS at the 2D limit in future studies.

\section{CONCLUSION}

In summary, we successfully synthesized quasi-vdW ferromagnet $\mathrm{Fe}_{0.26} \mathrm{TaS}_{2}$ single crystals and RBS/C measurement revealed that $\mathrm{Fe}$ atoms were intercalated at ordered positions between $\mathrm{TaS}_{2}$ layers. We performed a comprehensive study of the critical behavior of magnetization in quasi-vdW crystal $\mathrm{Fe}_{0.26} \mathrm{TaS}_{2}$ around its second-order FM-PM phase transition asymptotic region. The critical exponents $\beta=0.459(6)$, $\gamma=1.205(11), \delta=3.69(1)$, and critical temperature $T_{C}=$ 100.7 K were determined with various techniques, including modified Arrott plots, the KF method, the Widom scaling law, and critical isotherm analysis. Moreover, the reliability of established critical exponents was strictly checked and they obey the scaling equation well, which confirms that these exponents are self-consistent and intrinsic to the material. Furthermore, through renormalization group theory analysis, we found the spin interaction in FTS is of the 3D Heisenberg $(\{d: n\}=\{3: 3\})$ type with long-range magnetic coupling and that the exchange interaction decays with distance as $J(r) \sim r^{-4.71}$. Considering its approved strong magnetocrystalline anisotropy, long-range magnetic interaction, and the ability to be mechanically exfoliated into nanosheets, FTS is expected to maintain its magnetic coupling down to the 2D limit and has great potential for spintronic applications.

\section{ACKNOWLEDGMENTS}

This work was supported by King Abdullah University of Science and Technology (KAUST), Office of Sponsored Research (OSR) under Awards No. CRF-2017-3427-CRG6 and No. CRF-2018-3717-CRG7. Support from the Ion Beam Center (IBC) at Helmholtz-Zentrum Dresden-Rossendorf (HZDR) is gratefully acknowledged.
[1] C. Gong, L. Li, Z. L. Li, H. W. Ji, A. Stern, Y. Xia, T. Cao, W. Bao, C. Z. Wang, Y. A. Wang, Z. Q. Qiu, R. J. Cava, S. G. Louie, J. Xia, and X. Zhang, Nature (London) 546, 265 (2017).

[2] B. Huang, G. Clark, E. Navarro-Moratalla, D. R. Klein, R. Cheng, K. L. Seyler, D. Zhong, E. Schmidgall, M. A. McGuire, D. H. Cobden, W. Yao, D. Xiao, P. Jarillo-Herrero, and X. D. Xu, Nature (London) 546, 270 (2017).
[3] Z. Y. Fei, B. Huang, P. Malinowski, W. B. Wang, T. C. Song, J. Sanchez, W. Yao, D. Xiao, X. Y. Zhu, A. F. May, W. D. Wu, D. H. Cobden, J. H. Chu, and X. D. Xu, Nat. Mater. 17, 778 (2018).

[4] Y. J. Deng, Y. J. Yu, Y. C. Song, J. Z. Zhang, N. Z. Wang, Z. Y. Sun, Y. F. Yi, Y. Z. Wu, S. W. Wu, J. Y. Zhu, J. Wang, X. H. Chen, and Y. B. Zhang, Nature (London) 563, 94 (2018). 
[5] M. Bonilla, S. Kolekar, Y. J. Ma, H. C. Diaz, V. Kalappattil, R. Das, T. Eggers, H. R. Gutierrez, M. H. Phan, and M. Batzill, Nat. Nanotechnol. 13, 289 (2018).

[6] N. D. Mermin and H. Wagner, Phys. Rev. Lett. 17, 1133 (1966).

[7] B. K. Banerjee, Phys. Lett. 12, 16 (1964).

[8] Y. Liu and C. Petrovic, Phys. Rev. B 97, 014420 (2018).

[9] Y. Liu, V. N. Ivanovski, and C. Petrovic, Phys. Rev. B 96, 144429 (2017).

[10] B. Liu, Y. Zou, L. Zhang, S. Zhou, Z. Wang, W. Wang, Z. Qu, and Y. Zhang, Sci. Rep. 6, 33873 (2016).

[11] Y. Liu and C. Petrovic, Phys. Rev. B 96, 054406 (2017).

[12] Y. Liu and C. Petrovic, Phys. Rev. Mater. 3, 014001 (2019).

[13] S. S. P. Parkin and R. H. Friend, Philos. Mag. B 41, 65 (1980).

[14] N. J. Ghimire, A. Botana, J. Jiang, J. Zhang, Y.-S. Chen, and J. Mitchell, Nat. Commun. 9, 3280 (2018).

[15] T. Moriya and T. Miyadai, Solid State Commun. 42, 209 (1982).

[16] Y. Togawa, T. Koyama, K. Takayanagi, S. Mori, Y. Kousaka, J. Akimitsu, S. Nishihara, K. Inoue, A. S. Ovchinnikov, and J. Kishine, Phys. Rev. Lett. 108, 107202 (2012).

[17] H. Han, L. Zhang, D. Sapkota, N. Hao, L. Ling, H. Du, L. Pi, C. Zhang, D. G. Mandrus, and Y. Zhang, Phys. Rev. B 96, 094439 (2017).

[18] E. M. Clements, R. Das, L. Li, P. J. Lampen-Kelley, M.-H. Phan, V. Keppens, D. Mandrus, and H. Srikanth, Sci. Rep. 7, 6545 (2017).

[19] K.-T. Ko, K. Kim, S. B. Kim, H.-D. Kim, J.-Y. Kim, B. I. Min, J.-H. Park, F.-H. Chang, H.-J. Lin, A. Tanaka, and S.-W. Cheong, Phys. Rev. Lett. 107, 247201 (2011).

[20] E. Morosan, H. W. Zandbergen, L. Li, M. Lee, J. G. Checkelsky, M. Heinrich, T. Siegrist, N. P. Ong, and R. J. Cava, Phys. Rev. B 75, 104401 (2007).

[21] A. K. Pramanik and A. Banerjee, Phys. Rev. B 79, 214426 (2009).

[22] See Supplemental Materials at http://link.aps.org/supplemental/ 10.1103/PhysRevMaterials.3.114403 for the energy-dispersive $\mathrm{x}$-ray spectrum and atomic force microscope images of FTS samples.

[23] Y.-B. Qiao, Y.-L. Li, G.-H. Zhong, Z. Zeng, and X.-Y. Qin, Chin. Phys. 16, 3809 (2007).

[24] C. Riekel, H. G. Reznik, R. Schöllhorn, and C. J. Wright, J. Chem. Phys. 70, 5203 (1979).

[25] J. Dijkstra, P. J. Zijlema, C. F. Vanbruggen, C. Haas, and R. A. Degroot, J. Phys.: Condes. Matter 1, 6363 (1989).

[26] M. Mayer, SIMNRA user's guide, Report IPP 9/113 (MaxPlanck-Institut für Plasmaphysik, Garching, Germany, 1997).

[27] M. Mayer, in Application of Accelerators in Research and Industry, Pts 1 and 2, edited by J. L. Duggan and I. L. Morgan (AIP, Melville, 1999), pp. 541-544.
[28] J. I. Hoppe, J. Chem. Educ. 49, 505 (1972).

[29] H. Narita, H. Ikuta, H. Hinode, T. Uchida, T. Ohtani, and M. Wakihara, J. Solid State Chem. 108, 148 (1994).

[30] P. Rhodes and E. P. Wohlfarth, Proc. R. Soc. London Ser. A 273 , 247 (1963).

[31] E. P. Wohlfarth, J. Magn. Magn. Mater. 7, 113 (1978).

[32] M. Eibschütz, S. Mahajan, F. DiSalvo, G. Hull, and J. Waszczak, J. Appl. Phys. 52, 2098 (1981).

[33] C.-W. Chen, S. Chikara, V. S. Zapf, and E. Morosan, Phys. Rev. B 94, 054406 (2016).

[34] A. Arrott, Phys. Rev. 108, 1394 (1957).

[35] T. N. Lamichhane, L. Xiang, Q. Lin, T. Pandey, D. S. Parker, T.-H. Kim, L. Zhou, M. J. Kramer, S. L. Bud'ko, and P. C. Canfield, Phys. Rev. Mater. 2, 084408 (2018).

[36] A. Arrott and J. E. Noakes, Phys. Rev. Lett. 19, 786 (1967).

[37] M. E. Fisher, Rep. Prog. Phys. 30, 615 (1967).

[38] S. N. Kaul, J. Magn. Magn. Mater. 53, 5 (1985).

[39] J. C. Le Guillou and J. Zinn-Justin, Phys. Rev. B 21, 3976 (1980).

[40] D. Kim, B. Revaz, B. L. Zink, F. Hellman, J. J. Rhyne, and J. Mitchell, Phys. Rev. Lett. 89, 227202 (2002).

[41] J. S. Kouvel and M. E. Fisher, Phys. Rev. 136, A1626 (1964).

[42] B. Widom, J. Chem. Phys. 43, 3898 (1965).

[43] H. E. Stanley, Introduction to Phase Transitions and Critical Phenomena (Oxford University Press, London, 1971).

[44] M. Phan, V. Franco, N. Bingham, H. Srikanth, N. Hur, and S. Yu, J. Alloy. Compd. 508, 238 (2010).

[45] A. Taroni, S. T. Bramwell, and P. C. Holdsworth, J. Phys.: Condes. Matter 20, 275233 (2008).

[46] B. Liu, Y. Zou, S. Zhou, L. Zhang, Z. Wang, H. Li, Z. Qu, and Y. Zhang, Sci. Rep. 7, 6184 (2017).

[47] Q. Mao, B. Chen, J. Yang, Y. Zhang, H. Wang, and M. Fang, J. Phys.: Condes. Matter 30, 345802 (2018).

[48] Y. Liu and C. Petrovic, Phys. Rev. B 98, 064423 (2018),

[49] M. A. McGuire, H. Dixit, V. R. Cooper, and B. C. Sales, Chem. Mater. 27, 612 (2015).

[50] G. T. Lin, H. L. Zhuang, X. Luo, B. J. Liu, F. C. Chen, J. Yan, Y. Sun, J. Zhou, W. J. Lu, P. Tong, Z. G. Sheng, Z. Qu, W. H. Song, X. B. Zhu, and Y. P. Sun, Phys. Rev. B 95, 245212 (2017).

[51] M. E. Fisher, S.-k. Ma, and B. Nickel, Phys. Rev. Lett. 29, 917 (1972).

[52] S. Fischer, S. N. Kaul, and H. Kronmüller, Phys. Rev. B 65, 064443 (2002).

[53] D. L. Cortie, G. L. Causer, K. C. Rule, H. Fritzsche, W. Kreuzpaintner, and F. Klose, Adv. Funct. Mater., 1901414 (2019). 